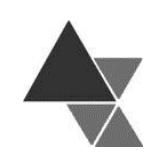

SEGURANCA

alimentar e nutricional

\title{
Portaria CVS no 5/2013 como ferramenta de prevenção e controle de doenças transmitidas por alimentos
}

\author{
Luana Limoeiro Ferrão ${ }^{1}$, Isabela Cristina Lobo de Morais ${ }^{2}$ e Elisa Helena da Rocha Ferreira ${ }^{3}$
}

A vigilância em saúde possui como objetivo a observação e análise constantes da situação de saúde da população. As doenças transmitidas por alimentos e seus surtos são um dos maiores desafios para a Saúde Pública. Estes surtos devem ser notificados, segundo o Sistema Nacional de Vigilância Epidemiológica vigente, sendo que, paralelamente à investigação, devem ser tomadas medidas de prevenção e controle. No Brasil esse perfil epidemiológico ainda é pouco conhecido, presumindo-se alta morbidade sem conhecer sua real magnitude. Buscando diminuir os casos destes surtos, a Secretaria do Estado de São Paulo aprovou em abril de 2013 a Portaria CVS no 5. Neste regulamento foram acrescidas medidas de prevenção e controle importantes para assegurar a diminuição do risco de contaminação do alimento. Este trabalho teve como objetivo discutir os avanços da legislação em relação à segurança dos alimentos, analisando as diretrizes da nova Portaria CVS no 5/2013. Constatou-se que é uma legislação mais rígida e detalhada que as revogadas CVS no 6/1999 e CVS no 18/2008 e até mesmo que a legislação federal em vigor, $\mathrm{RDC}$ no $216 / 2004$, principalmente no que tange a saúde e segurança do manipulador, processamento e armazenamento dos alimentos.

Palavras-chave: legislação sanitária, doenças transmitidas por alimentos, vigilância epidemiológica.

\section{Ordinance CVS 5/2013 as a tool of prevention and control of foodborne disease}

Health surveillance aims the constant observation and analysis of the population health condition. Foodborne disease and its outbreaks are one of the greatest challenges for Public Health. Those outbreaks must be notified, according to the National System of Epidemiologic Vigilance, and prevention and control actions must be taken simultaneously to the investigation. In Brazil, this epidemiological profile is not well known, assuming high morbidity without understanding its real magnitude., The Secretary of São Paulo state sanctioned in April 2013 the Ordinance CVS 5, seeking to decrease the cases of foodborne disease outbreaks. In this regulation, important measures were included to prevent and control the risk of food contamination. This study aimed to discuss the progress of legislation in relation to food safety, analyzing the guidelines of the new Ordinance CVS 5/2013. It was found that it is a more rigid and detailed legislation when compared to CVS 6/1999 and CVS 18/2008, and even the federal legislation RDC 216/2004, especially regarding the health and safety of the handler, food processing and storage.

Keywords: sanitary legislation, foodborne illnesses, epidemiological vigilance.

\footnotetext{
${ }^{1}$ Professora da Universidade Estácio de Sá. Endereço para correspondência: Rua André Rocha no 838, Taquara, CEP 22710-560, Rio de Janeiro - RJ. E-mail: luana.limoeiro.ferrao@gmail.com

${ }^{2}$ Nutricionista Pleno da Gerência Corporativa de Saúde da Petrobrás. Av. Almirante Barroso, nº 08, 23ํo andar, Centro, CEP 20031-045, Rio de Janeiro - RJ.

${ }^{3}$ Professora do Programa de Pós-Graduação em Ciência e Tecnologia de Alimentos. Instituto de Tecnologia, Departamento de Tecnologia de Alimentos, Universidade Federal Rural do Rio de Janeiro. Endereço para correspondência: Rodovia BR 465, km 7, CEP 23890-000, Seropédica - RJ.
} 


\section{INTRODUÇÃO}

Fazer Vigilância em Saúde é um desafio que exige muito planejamento e convergência de esforços do setor público e privado. Segundo o Ministério da Saúde ${ }^{[1]}$ o objetivo da Vigilância em Saúde engloba a observação e análise constantes da situação de saúde da população. Sob este aspecto é imprescindível a articulação entre políticas públicas, legislações e fiscalização para controlar determinantes, riscos e danos à saúde da população.

A Vigilância Epidemiológica possui a finalidade de recomendação e adoção de medidas, com o intuito de prevenir e controlar doenças e agravos. Determina assim o conjunto de ações para proporcionar o conhecimento, detecção ou prevenção de qualquer mudança que venha a ocorrer nos fatores determinantes e condicionantes do estado de saúde, seja individual ou coletiva ${ }^{[2]}$.

Entende-se por Doenças Transmitidas por Alimentos (DTA) aquelas causadas pela ingestão de alimentos ou bebidas contaminadas. A definição de surto é a ocorrência de dois ou mais casos epidemiologicamente relacionados à ingestão de alimentos e/ou água da mesma origem ${ }^{[2,3]}$. Estas DTA e surtos devem ser notificados, segundo a recomendação do Sistema Nacional de Vigilância Epidemiológica vigente, sendo que, paralelamente à investigação devem ser tomadas medidas de prevenção e controle. Nesta perspectiva, os dados dos surtos precisam estar disponíveis tanto para os que lideram as ações de políticas públicas quanto para a população em geral [2].

As DTA e os surtos, de forma geral, são eventos muitas vezes graves, súbitos e de curta duração. É essencial o rápido e adequado planejamento das atividades a serem desenvolvidas, logo que se tenha o conhecimento da suspeita, atentando-se especificamente para coleta de amostras e dados para que devidas medidas de prevenção e controle sejam mais eficazes com o objetivo de se evitar a ocorrência de novos surtos ${ }^{[4]}$.

Nos Estados Unidos é calculada a carga de DTA como ferramenta de definição de prioridades para o desenvolvimento de políticas, pesquisa, educação e legislação. A carga de doenças é um índice mensurável, obtido através de uma complexa fórmula, a qual utiliza variáveis como anos de vida perdido por morte prematura $(Y L L)$, anos de vida perdido por incapacidade $(Y L D)$, além de mortalidade, prevalência, esperança de vida e peso das incapacidades-em consideração e estima o número de casos de DTA que existem na comunidade, incluindo casos reportados e não reportados. O Centro de Controle e Prevenção de Doenças (Centers for Disease Control and Prevention - CDC) usará esta estimativa para atribuir doenças a fontes de alimentos e determinar os custos de DTA ${ }^{[5,6]}$. Estima-se que $1 \mathrm{em}$ cada 6 americanos ficarão doentes, 128.000 serão hospitalizados e 3.000 chegarão a óbito por ano, sendo o custo econômico anual, apenas para o governo americano, de aproximadamente 77 bilhões de dólares ${ }^{[7] .}$ Apesar desta estimativa a subnotificação dos surtos de DTA ainda é um problema ${ }^{[6]}$. Scallan et al. ${ }^{[5]}$ concluíram que $90 \%$ das doenças são causados por patógenos conhecidos, sendo eles, Salmonella, Norovirus, Campylobacter, Toxoplasma, Escherichia coli O157, Listeria e Clostridium perfringens.

No Brasil esse perfil epidemiológico ainda é pouco conhecido. Presume-se alta morbidade, entretanto como poucas DTA estão incluídas no Sistema Nacional de Vigilância Epidemiológica, não se conhece sua real magnitude ${ }^{[2]}$. Somente cerca de $10 \%$ do total de surtos de origem alimentar são notificados no Brasil devido a falhas no sistema de notificação e de fiscalização ${ }^{[8]}$.

Estudos mostram que muitas doenças são causadas por patógenos que podem ser controlados e que esta prevalência tende a declinar quando medidas preventivas e de controle são implementadas ${ }^{\left[{ }^{[-12]}\right.}$.

Com a finalidade de evitar estes surtos e obter alimentos que sejam seguros ao consumo, é imprescindível a adoção de medidas de controle do processo produtivo do alimento. Sob este aspecto, existem ferramentas que devem ser adotadas pelos produtores de alimentos durante a manipulação, a partir das Boas Práticas de Fabricação (BPF), dos Procedimentos Padrões Operacionais (POP) e pelo uso do Sistema de Análise de Perigos e Pontos Críticos de Controle (APPCC), as quais são de conhecida importância para a redução dos perigos de origem alimentar ${ }^{[11]}$. 
Prevenção e controle de doenças transmitidas por alimentos. Ferrão, Morais e Ferreira

Assim, os Órgãos Governamentais aprovam Regulamentos Técnicos de Boas Práticas para estabelecimentos comerciais de alimentos e serviços de alimentação, sendo, as mais importantes, no âmbito Federal a Resolução da Diretoria Colegiada no 216 de 15 de setembro de 2004 (RDC n- 216/2004) da Agência Nacional de Vigilância Sanitária (ANVISA) e na esfera do Estado de São Paulo a nova Portaria CVS no 5 de 19 de abril de $2013^{[3,13]}$.

Este trabalho teve como objetivo discutir os avanços da legislação em relação à segurança dos alimentos, analisando as diretrizes da nova Portaria CVS no 5/2013 como forma de prevenção e controle das doenças transmitidas por alimentos e compará-la com a Resolução da Diretoria Colegiada 216 de 15 de setembro de 2004 (RDC no 216/2004) da ANVISA e as Portarias CVS no ${ }^{\circ}$ 6/1999 e CVS no 18/2008 ${ }^{[14,15]}$.

\section{METODOLOGIA}

Foi realizado um levantamento das estatísticas dos surtos de DTA e números de doentes no Brasil decorrentes destes surtos junto aos dados oficiais da Coordenação de Vigilância das Doenças de Transmissão Hídrica e Alimentar, Secretaria de Vigilância em Saúde do Ministério da Saúde, no período de 2000 a $2013^{[16]}$.

Frente a publicação da nova Portaria CVS no 5 do Centro de Vigilância Sanitária da Secretaria de Estado da Saúde de São Paulo, no Diário Oficial do Estado de São Paulo em 19 de abril de 2013 que revoga as Portarias CVS no 6/1999 e CVS no 18/2008, foram destacadas e analisadas as diretrizes que contribuem para a redução da carga de DTA ${ }^{[3,14,15]}$.

Foram verificadas as diferenças entre todos os parâmetros desta nova Legislação Estadual de São Paulo e os preconizados na Resolução da Diretoria Colegiada 216 de 15 de setembro de 2004 (RDC n216/2004) da ANVISA, além das alterações em relação àquelas revogadas.

\section{RESULTADOS E DISCUSSÃO}

\section{Dados Epidemiológicos}

Os dados publicados, em relação ao número de surtos e doentes de DTA no Brasil, entre o período de 2000 a 2013, segundo a Secretaria de Vigilância em Saúde, do Ministério da Saúde, pode ser visualizado no Gráfico 1. Observase que desde 2000 foram notificados 8.860 surtos de doenças transmitidas por alimentos no Brasil com mais de 145.000 doentes. Em 2005, a Secretaria de Vigilância Sanitária publicou em boletim que no período de 1999 a 2004 o Governo Federal teve um custo de 280 milhões de reais com 3.737 surtos de DTA envolvendo aproximadamente 73.500 doentes ${ }^{[16]}$.

Gráfico 1. Número de surtos e doentes por DTA no Brasil, entre 2000 e 2013.

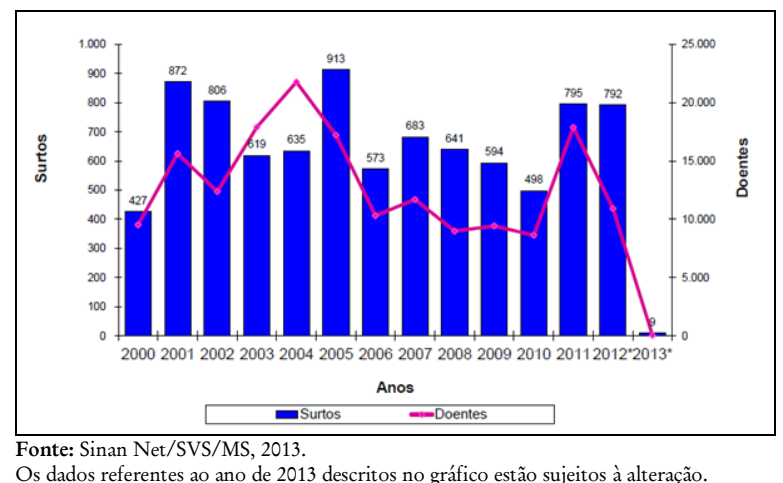

O Gráfico 2 mostra que os principais alimentos envolvidos nos surtos por DTA são alimentos mistos (28\%), ou seja, que possuem ingredientes de diferentes origens, como animal e vegetal e alimentos à base de ovos (15\%). Devido à manipulação que os alimentos mistos sofrem durante o preparo e à contaminação de ovos com Salmonella, estes acabam estando envolvidos em maior proporção nos surtos ${ }^{[12,17,18,19]}$.

A água foi responsável por $9 \%$ dos casos de surto, sendo o terceiro principal alimento responsável pelas DTA no Brasil. Devido ao seu consumo em larga escala, supõe-se que há subnotificação dos surtos causados por água contaminada. Mesmo assim, este não é um indicador favorável, tendo em vista o seu elevado consumo direto ou indiretamente, na higienização ou preparo de refeições. Os alimentos de origem animal e vegetal corresponderam a 20,5 e $2,5 \%$, respectivamente, e o pescado e derivados a $1,5 \%$ dos casos de surtos ${ }^{[19]}$. 
Prevenção e controle de doenças transmitidas por alimentos. Ferrão, Morais e Ferreira

Gráfico 2. Número de surtos de DTA por tipo de alimento no Brasil, entre 2000 e 2013.

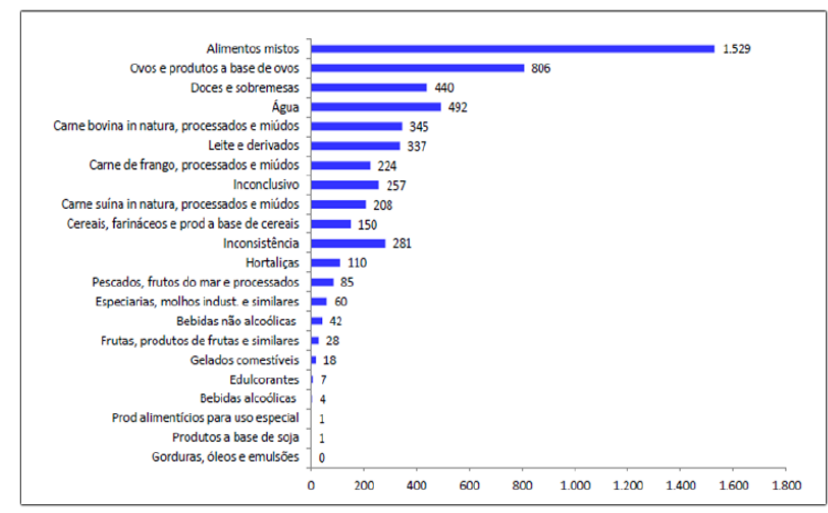

Fonte: Sinan Net/SVS/MS, 2013.

Inconsistência - Informações não condizentes com o campo de preenchimento no momento do registro do surto.

Inconclusivo - Quando o campo apresentou informações vagas.

Buscando diminuir os casos de surtos por DTA, a Secretaria do Estado de São Paulo aprovou em abril de 2013 a Portaria CVS n- 5/2013, a qual revoga a Portaria CVS no 6/1999 e a Portaria CVS no $18 / 2008$, o regulamento técnico sobre boas práticas. Neste regulamento foram acrescidas medidas que assegurem a diminuição do risco de contaminação do alimento, além de medidas de segurança do trabalho para o manipulador de alimentos.

\section{Controle de Saúde dos Manipuladores}

Em relação ao controle de saúde dos funcionários foi mantida a exigência dos exames exigidos pelo Programa de Controle Médico de Saúde Ocupacional (PCMSO) e o Atestado de saúde Ocupacional (ASO). Estes deverão ser mantidos no local de trabalho para comprovação junto às autoridades sanitárias no momento da fiscalização.

Os funcionários com qualquer doença e/ou lesão que leve ao risco de contaminação do alimento, incluindo as infecções oculares e as orofaríngeas, deverão ser afastados da atividade de manipulação de alimentos enquanto persistir estas condições de saúde. A inclusão destas duas patologias citadas está relacionada, principalmente, à contaminação por Staphylococcus aureus subsp. aureus, considerado o principal agente causador de surtos de doenças transmitidas por alimentos [20], micro-organismo presente nas mucosas de seres humanos, principalmente na orofaríngea. Estima-se que de 20 a
$40 \%$ de humanos adultos são portadores assintomáticos, apresentando este micro-organismo nas fossas nasais ${ }^{[21]}$.

A CVS no 5/2013 aborda as condições de higiene e segurança dos funcionários. Foi acrescida, no asseio dos funcionários, a obrigatoriedade de raspar bigodes e barbas diariamente e o piercing como adorno que deve ser retirado para a manipulação dos alimentos. Os uniformes não possuem mais a obrigatoriedade de cor branca, mas os cabelos deverão estar presos e totalmente protegidos e os sapatos deverão ser fechados e antiderrapantes. É permitido guardar objetos no bolso inferior do uniforme, desde que estes sejam para uso no local de trabalho, como, por exemplo, caneta, lápis e termômetro. Os equipamentos de proteção individual (EPI) deverão ser utilizados em câmaras frias, cortes de carnes, situação de calor intenso, manipulação de saneantes, lixo e na lavagem de sanitários. No Brasil, o Ministério do Trabalho regulamenta, através da NR6, a questão do uso de EPI pelos funcionários ${ }^{[22]}$.

A nova recomendação é em relação à proibição de máscara nasobucal devido à ausência de comprovação de eficácia em relação ao seu uso. $\mathrm{Na}$ realidade a utilização das máscaras durante a manipulação de alimentos pode provocar efeito inverso, pois a máscara de pano ou a de fibra descartáveis provoca uma maior contaminação porque após 15 a 30 minutos de uso ocorre a umidificação das fibras e abre espaços virtuais, facilitando a passagem de gotículas de saliva. Além desse fator, o abafamento causado pela máscara nas narinas acumula $\mathrm{CO}_{2}$ (gás carbônico), gás este irritante às mucosas, provocando aceso de tosse e, como consequência, maior contaminação. Ocorre também prurido no nariz do manipulador, fazendo assim com que haja o contato da mão com as narinas, aumentando assim a contaminação dos dedos com $S$. aureus. Por fim, o abafamento decorrente do uso da máscara provoca retenção de micro-organismos no trato respiratório, aumentando a possibilidade de ocorrência de infecções pulmonares ${ }^{[12]}$.

A antissepsia das mãos deverá ser efetuada de forma frequente, principalmente antes de utilizar utensílios higienizados e luvas descartáveis. Para este procedimento deve ser utilizado sabonete líquido, neutro, inodoro e com ação antisséptica e o mesmo 
deverá durar 3 minutos para que seja garantida uma ação eficiente deste processo. Cartazes com o correto procedimento de lavagem deverão ser afixados nas pias exclusivas para este fim. Esse procedimento tem como finalidade eliminar a microbiota transitória da pele e reduzir a microbiota residente, além de proporcionar efeito residual na pele do manipulador [23].

\section{Manipulação dos Alimentos}

Durante a recepção das mercadorias deverão ser observadas as características quantitativas, qualitativas e sensoriais das matérias-primas. Uma grande diferença entre a CVS no 5/2013 e a CVS no 6/1999 está em relação à temperatura de recebimento dos alimentos congelados e refrigerados, temperatura esta que deverá ser conferida e registrada no momento da recepção. Estas temperaturas podem ser observadas na Tabela 1. Na RDC no 216/2004 não existe nenhuma especificação quanto à temperatura no ato de recebimento de mercadorias, apenas que deve ser obedecido o recomendado pelo fabricante.

Tabela 1. Temperatura de recebimento de alimentos congelados e refrigerados.

\begin{tabular}{c|c|c}
\hline Tipo de Alimento & $\begin{array}{c}\text { CVS no 6/1999 } \\
\text { Temperatura em }{ }^{\circ} \mathrm{C}\end{array}$ & $\begin{array}{c}\text { CVS no } 5 / 2013 \\
\text { Temperatura em }{ }^{\circ} \mathrm{C}\end{array}$ \\
\hline Congelados & -12 a -18 & $\begin{array}{c}-12 \\
\text { Pescados } \rightarrow 2 \text { a } 3 \text { ou CRF* }\end{array}$ \\
Refrigerados & & $\begin{array}{c}\text { Carnes } \rightarrow \text { a } 7 \text { ou CRF* } \\
\text { Demais produtos } \rightarrow \text { a } 10 \text { ou CRF* }\end{array}$ \\
Resfriados & 6 com tolerância de 7 & Não possui essa designação \\
\hline *CRF - Conforme Recomendação do Fabricante. & 6 a 10 ou CRF*
\end{tabular}

Em relação ao armazenamento de produtos, é permitido o uso de caixas de madeira apenas para os peixes salgados e algumas frutas que venham embaladas neste tipo de material, desde que estejam dispostas em uma área exclusiva para o seu armazenamento, e para todos os outros alimentos o seu uso na área de armazenamento é proibido. A caixa de papelão pode permanecer sob refrigeração ou congelamento, desde que em local delimitado ou equipamento exclusivo e não deve apresentar umidade e bolores. Estas embalagens podem adentrar a área de manipulação, desde que seja evitada a contaminação cruzada e as mesmas estejam limpas. Sempre que possível as embalagens deverão ser lavadas antes de sua abertura para uso.
Para os alimentos fracionados ou retirados da embalagem original deverá ser mantida a etiqueta original ou etiquetas próprias contendo as informações do produto, inclusive a data da transferência. A validade dos alimentos e a temperatura de armazenamento recomendadas podem ser visualizadas na Tabela 2. A CVS no 5/2013 acrescenta a proibição da prática de desligar o refrigerador com o objetivo de economizar energia e o uso de termômetro de haste de vidro para controlar a temperatura, visando assim a qualidade dos produtos refrigerados. 
Prevenção e controle de doenças transmitidas por alimentos. Ferrão, Morais e Ferreira

Tabela 2. Validade e temperatura de armazenamento para alimentos refrigerados e congelados.

\begin{tabular}{lcc}
\hline \multicolumn{1}{c}{ Alimento } & Temperatura $\left({ }^{\circ} \mathrm{C}\right)$ & Dias \\
\hline Pescados e seus produtos manipulados crus & Máximo 2 & 3 \\
Pescados pós-cocção & Máximo 2 & 1 \\
Alimentos pós-cocção, exceto pescado & Máximo 4 & 3 \\
Carnes bovina, suína, aves, entre outras, e seus produtos manipulados crus & Máximo 4 & 3 \\
Espetos mistos, bife role, carnes empanadas cruas e preparações com carne moída & Máximo 4 & 2 \\
Frios e embutidos fatiados, picados ou moídos & Máximo 4 & 3 \\
Maionese e misturas de maionese com outros alimentos & Máximo 4 & 2 \\
Sobremesas e outras preparações com laticínios & Máximo 4 & 3 \\
Demais alimentos preparados & Máximo 4 & 3 \\
Produtos de panificação, confeitaria com coberturas e recheios, prontos para o consumo & Máximo 4 & 5 \\
Frutas, verduras e legumes higienizados, fracionados ou descascados; sucos e polpas de frutas & Máximo 4 & 3 \\
Leite e derivados & Máximo 7 & 5 \\
Ovos & Máximo 10 \\
Alimentos Congelados & 0 a -5 & 7 \\
& -6 a -10 & 10 \\
\hline
\end{tabular}

O descongelamento dos alimentos que serão manipulados e/ou consumidos deverá seguir as recomendações do fabricante ou ocorrer sempre sob refrigeração, em temperatura inferior a $5^{\circ} \mathrm{C}$, quando for um descongelamento lento ou em micro-ondas quando for um descongelamento rápido, assim como recomenda a RDC no 216/2004. É proibido descongelar os alimentos a temperatura ambiente, diminuindo assim o risco de proliferação microbiana nos alimentos durante as faixas críticas de temperatura ${ }^{[12,17]}$. Essa alteração permitiu a adequação à legislação federal, uma vez que a CVS no 6/1999 permitia descongelar em água com temperatura inferior a $21^{\circ} \mathrm{C}$ por 4 horas e em temperatura ambiente, em local sem contaminação ambiental, monitorando a temperatura superficial até atingir 3 a $4^{\circ} \mathrm{C}$ e continuar o degelo sob refrigeração. Segundo a nova CVS no 5/2013 o alimento descongelado nunca pode ser recongelado.

O dessalgue deverá ser feito conforme recomendação do fabricante ou em água potável a uma temperatura inferior a $5^{\circ} \mathrm{C}$ ou em água sob fervura. Este é outro ponto de mudança em relação à CVS n- 6/1999 a qual permitia que este processo acontecesse com trocas de água à temperatura máxima de $21^{\circ} \mathrm{C}$ ou a cada 4 horas; em água sob refrigeração até $10^{\circ} \mathrm{C}$; ou através da fervura. $\mathrm{Na} \mathrm{RDC}$ no 216/2004 não existe nenhuma orientação quanto a esta etapa de manipulação dos alimentos.
Para o procedimento de higienização de hortifrutícolas a recomendação é que seja realizado em água potável, com produto desinfetante para alimentos registrado e permitido o uso pela ANVISA, seguindo a seguinte recomendação para diluição: 10 $\mathrm{mL}$ ou 1 colher de sopa rasa de hipoclorito na concentração de 2-2,5\% em $1 \mathrm{~L}$ de água potável ou 20 $\mathrm{mL}$ ou 2 colheres de sopa rasa de hipoclorito na concentração de $1 \%$ em $1 \mathrm{~L}$ de água potável. Devem ser afixadas instruções visíveis e compreensíveis sobre o correto método de higienização no local onde o mesmo ocorre.

No preparo dos alimentos deverá ser atingida a temperatura de segurança de $74^{\circ} \mathrm{C}$ no centro geométrico do alimento. Este critério de temperatura é mais rígido que o exigido pela legislação federal ${ }^{[13]}$, com o objetivo de garantir uma cocção eficiente, contribuindo para a eliminação de micro-organismos patogênicos. O resfriamento dos alimentos deve ocorrer em equipamentos, de forma a minimizar o risco de contaminação cruzada e a permanência em temperatura de crescimento microbiano, reduzindo de $60^{\circ} \mathrm{C}$ a $10^{\circ} \mathrm{C}$ em até 2 horas. A alteração neste binômio tempo-temperatura para um critério mais rígido buscou utilizar a mesma diretriz da RDC n216/2004 para este aspecto evitando conflitos como acontecia entre a CVS n- 6/1999 e a legislação federal no tocante a resfriamento rápido de alimentos ${ }^{[13]}$. A CVS no 5/2013 traz detalhamento sobre o uso de ovos no preparo dos alimentos vista sua estreita 
Prevenção e controle de doenças transmitidas por alimentos. Ferrão, Morais e Ferreira

relação com surtos de DTA ${ }^{[19]}$. São proibidas preparações onde os mesmos estejam crus ou mal cozidos, sendo que o ovo cozido deverá ser fervido por 7 minutos e ovo frito servido com a gema dura. Em preparações sem cocção (maionese, mousse, entre outras) usar ovo pasteurizado, desidratados ou cozidos. Durante a manipulação do ovo, o mesmo deverá ocorrer de modo que o conteúdo não entre em contato com a casca. A fim de evitar a contaminação destas preparações, é proibido usar ovos com cascas rachadas e sujas. O armazenamento dos ovos deverá ser, preferencialmente, sob refrigeração, sendo recomendável lavá-los apenas antes do uso.

$\mathrm{Na}$ distribuição dos alimentos prontos ao consumo os mesmos devem estar expostos protegidos contra poeiras, insetos e outras pragas e contra contaminantes oriundos dos consumidores (gotículas de saliva, cabelo, entre outros) e distantes de produtos químicos e da incidência direta do fluxo de ar. Os ornamentos da área de distribuição não devem estar entre o fluxo de ar e os alimentos, tampouco sobre os balcões de distribuição. $\mathrm{O}$ recebimento de dinheiro e afins deverá ocorrer em área específica, por um funcionário que não manipule alimento, evitando assim a contaminação dos alimentos. Nos casos em que são utilizados balcões térmicos, a água deverá ser trocada diariamente, mantida entre $80-90^{\circ} \mathrm{C}$ e sua temperatura deverá ser aferida e registrada durante a distribuição. Deverá ser respeitado o binômio tempo e temperatura, conforme a Tabela 3. Caso não seja cumprido estes parâmetros o alimento exposto deverá ser descartado. É permitida a reutilização de alimentos para fins de doação gratuita, incluindo-se sobras, em qualquer etapa da produção, desde que elaborados com as Boas Práticas. Não incluem neste âmbito os restos dos pratos dos consumidores. A RDC n- 216/2004 recomenda apenas a exposição de alimentos quentes, os quais devem ser mantidos a uma temperatura superior a $60^{\circ} \mathrm{C}$ por no máximo 6 horas.

Tabela 3. Diferença entre a CVS no 5/2013 e a CVS no 6/1999 no binômio tempo versus temperatura a ser respeitado na distribuição dos alimentos.

\begin{tabular}{c|c|c}
\hline \multirow{2}{*}{ Tipo de Alimento } & \multicolumn{2}{|c}{ Temperatura $\left({ }^{\circ} \mathrm{C}\right) x$ Tempo de Exposição (horas) } \\
\cline { 2 - 3 } & $\mathrm{CVS} \mathrm{n}-5 / 2013$ & $\mathrm{CVS} \mathrm{n}^{\circ} 6 / 1999$ \\
\hline \multirow{2}{*}{ Alimentos Quentes } & $\geq 60^{\circ} \mathrm{C} /$ máximo 6 horas & $\geq 65^{\circ} \mathrm{C} / 12$ horas \\
& $<60^{\circ} \mathrm{C} /$ máximo 1 hora & $60^{\circ} \mathrm{C} / 6$ horas \\
& & $<60^{\circ} \mathrm{C} / 3$ horas \\
Alimentos Frios & Até $10^{\circ} \mathrm{C} /$ máximo 4 horas & Até $10^{\circ} \mathrm{C} / 4$ horas \\
& Entre $10 \mathrm{e} 21^{\circ} \mathrm{C} /$ máximo 2 horas & Entre $10 \mathrm{e} 21^{\circ} \mathrm{C} / 2$ horas \\
\hline
\end{tabular}

A guarda de amostra de alimentos servidos continuou sendo obrigatória, sendo que deve ser colhido na segunda hora do tempo de distribuição, com os mesmos utensílios usados para a distribuição. As mesmas devem ser mantidas sob refrigeração a no máximo $4^{\circ} \mathrm{C}$, no caso de alimentos refrigerados e congelados a $-18^{\circ} \mathrm{C}$ no caso de alimentos quentes, por 72 horas, tempo máximo de aparecimento de algum surto relacionado à DTA.

Diferentemente da CVS no 6/1999 que descrevia, detalhadamente, a higiene ambiental em relação à periodicidade, etapas obrigatórias e produtos que podem ser utilizados, a CVS n- 5/2013 determina que a periodicidade e os procedimentos para higienização devem ser estabelecidos em procedimentos operacionais padronizados (POP). Esta nova legislação aponta a preocupação com a segurança do manipulador, sinalizando que nesta etapa os manipuladores devem utilizar EPI, como luvas nitrílicas de cano longo, óculos e bota de borracha, sendo sempre obedecidas as instruções de uso e de segurança recomendada pelo fabricante dos produtos. A RDC n- 216/2004 determina apenas que a área de preparação deve ser higienizada quantas vezes forem necessárias e imediatamente após o término do trabalho, ressaltando que um POP que deve ser implantado, segundo recomendação desta lei 
federal, é relacionado à higienização de instalações, equipamentos e móveis.

A descrição das áreas físicas não apresenta nenhuma diferença significativa entre a CVS $\mathrm{n}$ 5/2013, a CVS n- 6/1999 e a RDC n- 216/2004.

Em relação à caixa de gordura, a mesma deve ser localizada fora da área de manipulação e armazenamento de alimentos e ser limpa periodicamente.

\section{Potabilidade da Água}

A água utilizada no preparo dos alimentos deve ser proveniente de abastecimento público, devendo a mesma ser tratada e a qualidade controlada por análise laboratorial na periodicidade determinada pela legislação específica vigente. Essa determinação segue o recomendado pela RDC no 216/2004. No Brasil, a legislação federal que dispõe sobre os procedimentos de controle e de vigilância da qualidade da água para consumo humano e seu padrão de potabilidade é a portaria no $2914 / 2011^{[24]}$.

\section{Roteiro de Inspeção}

A CVS no 5/2013 apresenta, como anexo, um roteiro de inspeção das boas práticas em estabelecimentos comerciais de alimentos e serviço de alimentação, sendo uma ferramenta importante para a elaboração do relatório de inspeção de auditorias. Aplicando essa ferramenta o estabelecimento inspecionado consegue estabelecer um plano de ações corretivas com prazos de curto, médio e longo prazo para resolução das não-conformidades contribuindo para diminuir o risco de DTA ${ }^{[25]}$.

\section{CONCLUSÃO}

O registro e controle de DTA são um problema de saúde pública no Brasil. Os órgãos governamentais, a partir de publicação de novos padrões legais de exigência em relação às boas práticas de fabricação de alimentos, possuem papel de suma importância neste processo.

Constatou-se que a CVS no 5/2013 é uma legislação mais rígida e detalhada que as revogadas CVS no 6/1999 e CVS no 18/2008 e até mesmo que a legislação federal em vigor a RDC no 216/2004, principalmente no que tange a saúde e segurança do manipulador, processamento e armazenamento dos alimentos.

Sugere-se que a legislação federal seja complementada pelas diretrizes especificadas na CVS no 5/2013, o que pode levar a um impacto na redução da carga e nos custos de DTA no Brasil. Além disso, percebe-se a importância do acompanhamento na implementação desta nova legislação a fim de mensurar os possíveis impactos positivos no controle de surtos de DTA.

\section{REFERÊNCIAS}

[1] Ministério da Saúde (BR). Secretaria de Vigilância em Saúde, Secretaria de Atenção à Saúde. Diretrizes Nacionais da Vigilância em Saúde. Brasília: Ministério da Saúde; 2010.

[2] Ministério da Saúde (BR). Secretaria de Vigilância em Saúde. Manual Integrado de Prevenção e Controle de Doenças Transmitidas por Alimentos. Brasília: Ministério da Saúde; 2010.

[3] São Paulo (Estado). Secretaria de Saúde. Coordenação dos Institutos de Pesquisa. Centro de Vigilância Sanitária. Portaria CVS nํ 5, de 9 de abril de 2013. Aprova o regulamento técnico sobre boas práticas para estabelecimentos comerciais de alimentos e para serviços de alimentação, e o roteiro de inspeção, anexo. Diário Oficial do Estado de São Paulo. 19 abr 2013.

[4] Ministério da Saúde (BR). Secretaria de Vigilância em Saúde, Departamento de Vigilância Epidemiológica. Guia de Vigilância Epidemiológica. 6. ed. Brasília: Ministério da Saúde; 2007.

[5] Scallan E, Hoekstra RM, Angulo FJ, Tauxe RV, Widdowson MA, Roy SL, Jones JL, Griffin PM. Foodborne Illness Acquired in the United States Major Pathogens. Emerg. Infect. Dis. 2011:17(1):7-15. DOI http://dx.doi.org/10.3201/eid1701.P11101.

[6] Morris JG. How Safe Is Our Food? Emerg. Infect. Dis. 2011; 17(1): 126-128. DOI http://dx.doi.org/10.3201/eid1701101821.

[7] Centers for Disease Control and Prevention (CDC). Estimates of Foodborne Illness in the United States 
Prevenção e controle de doenças transmitidas por alimentos. Ferrão, Morais e Ferreira

[internet]. Atlanta; 2013 [acesso em 11 mai 2013]. Disponível em: http://www.cdc.gov/foodborneburden

[8] Shinohara NKS, Barros VB, Jimenez SMC, Machado ECL, Dutra RAF, Lima Filho JL. Salmonella spp., importante agente patogênico veiculado em alimentos. Ciência \& Saúde Coletiva. 2008;13(5):1675-1683.

[9] Sarter G, Sarter S. Promoting a culture of food safety to improve hygiene in small restaurants in Madagascar. Food Control. 2012;25:165-171.

[10] Arent SW, Paez P, Strohbehn C. Food safety practices and manager's perceptions: a qualitative study in hospitality. IJCHM. 2013;25(1):124-139.

[11] Ebone MV, Cavalli SB, Lopes SJ. Segurança e qualidade higiênico-sanitária em unidades produtoras de refeições comerciais. Rev. Nutr. 2011;24(5):725-734.

[12] Silva Júnior EA. Manual de Controle HigiênicoSanitário em Serviços de Alimentação. São Paulo: Editora Varela; 2013.

[13] Brasil. Ministério da Saúde. Agência Nacional de Vigilância Sanitária. Resolução da Diretoria Colegiada no 216, de 15 de setembro de 2004. Dispõe sobre regulamento técnico de boas práticas para serviços de alimentação. Diário Oficial da União. 16 set 2004.

[14] São Paulo (Estado). Secretaria de Saúde. Coordenação dos Institutos de Pesquisa. Centro de Vigilância Sanitária. Portaria CVS no 6, de 10 de março de 1999. Dispõe sobre regulamento técnico de parâmetros e critérios para o controle higiênico sanitário em estabelecimentos de alimentos. Diário Oficial do Estado de São Paulo. 12 de mar 1999.

[15] São Paulo (Estado). Secretaria de Saúde. Coordenação dos Institutos de Pesquisa. Centro de Vigilância Sanitária. Portaria CVS no 18 , de 11 de setembro de 2008. Aprova alteração do item 4 Controle de saúde dos funcionários, do item 16 Higiene Ambiental e do subitem 16.3 da Portaria CVS no 6/99. Diário Oficial do Estado de São Paulo. 11 de set 2008.

[16] Brasil. Ministério da Saúde. Secretaria de Vigilância em Saúde. Boletim Eletrônico Epidemiológico. Vigilância epidemiológica das doenças transmitidas por alimentos no Brasil, 1999 - 2004 [internet]. Brasília; 2005 [acesso em 25 mai 2013]. Disponível em: http://portalsaude.saude.gov.br

[17] Germano PML. Higiene e Vigilância Sanitária de Alimentos. São Paulo: Editora Manole; 2008.

[18] Barancelli GV, Martin JGP, Porto E. Salmonella em ovos: relação entre produção e consumo seguro. Segur. Aliment. Nutr. 2012;19(2):73-82.

[19] Brasil. Ministério da Saúde. Sistema de Informação de Agravos e Notificação (SINAN) [internet]. Brasília; 2013 [acesso em 11 mai 2013]. Disponível em: http://dtr2004.saude.gov.br/sinanweb

[20] Brandão MLL, Costa JCB, Farias FM, Rosas CO, Bricio SML, Nascimento JS, Cardarelli-Leite P. Desenvolvimento de material de referência para microbiologia de alimentos contendo estafilococos coagulase positiva em matriz queijo. Braz. J. Food Technol. 2013;16(1):73-79.

[21] Cunha AP. Presença de Staphylococcus coagulase positiva em queijo minas frescal artesanal [dissertação]. Minas Gerais: Universidade Federal de Uberlândia; 2012.

[22] Brasil. Ministério do Trabalho e Emprego. Portaria n- 3.214, de 08 de junho de 1978. Aprova as normas regulamentadoras que consolidam as leis do trabalho, relativas à segurança e medicina do trabalho; NR - 6 e Equipamento de Proteção Individual - EPI. Diário Oficial da União. 06 jul 1978.

[23] Brasil. Ministério da Saúde. Agência Nacional de Vigilância Sanitária. Anti-sepsia cirúrgica ou preparo pré-operatório das mãos [internet]. Brasília, 2013 [acesso em 14 mai 2013]. Disponível em: http://www.anvisa.gov.br

[24] Brasil, Ministério da Saúde. Portaria no 2.914, de 12 de dezembro de 2011. Dispõe sobre os procedimentos de controle e de vigilância da qualidade da água para consumo humano e seu padrão de potabilidade. Diário Oficial da União. 14 dez 2011. Seção 1.

[25] Morais ICL, Costa SRR. Proposta de ferramentas de qualidade para um sistema de gestão de segurança de alimentos em Unidades de Alimentação e Nutrição, Alim. Nutr. 2013;24(1):16-20. 